\title{
Productivity and Impact of the Top 100 Cited Parkinson's Disease Investigators since 1985
}

\author{
Aaron A. Sorensen ${ }^{\mathrm{a}, *}$ and David Weedon ${ }^{\mathrm{b}}$

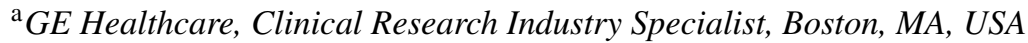 \\ ${ }^{\mathrm{b}}$ Publishing Consultant, London, UK
}

\begin{abstract}
We have compiled a list of the 100 most cited researchers in Parkinson's disease since 1985 together with H-Indices as a means to assess productivity and impact. Within the total-citations ranking, "broad impact" citations are used as a way of identifying those researchers whose work is cited widely beyond the Parkinson's disease research community. Finally, we present a table of the most cited researchers this decade for a comparison of the two with analysis.
\end{abstract}

Keywords: Parkinson's disease, neurodegenerative diseases, neurosciences, bibliometrics, scientometrics, H-index, authorship, factual databases, ranking, citation, citation analysis, highly-cited, history of science

\section{INTRODUCTION}

The number of citations an article receives is widely accepted as a measure of its impact. In recent years, the field of Parkinson's Disease (PD) research has been the focus of two bibliometric studies in which the mosthighly cited papers were identified [1] and a partial ranking of top authors was generated [2]. There has not, however, been a broad analysis of the PD research literature to assess, in a comprehensive manner, the impact and productivity of the top investigators, which this study aims to provide.

\section{METHODS}

The following three dimensions were selected as a basis to measure the work in PD by individual

${ }^{*}$ Correspondence to: Aaron A. Sorensen, GE Healthcare, Clinical Research Industry Specialist, 116 Huntington Avenue, Boston, MA 02116, USA. E-mail: aaron.sorensen@ge.com. investigators: total citations, "broad impact" citations and $\mathrm{H}$-index. Briefly, the H-index is a measure of an author's highly cited body of work rather than of individual papers - for details see [3, 4]. Broad impact citations are a new measure, discussed below. The underlying data used in the tabulation of each dimension originate from Thomson Reuter's ISI Web of Science (WoS).

Two selection filters were used in determining which papers would contribute to an individual scientist's metrics. The first filter was a requirement that all papers to be included in the analysis mention "Parkinson," "Parkinson's," "Parkinsons," "Parkinsonism," or "Parkinsonian" in the title while excluding those papers which contained the term "amyotrophic" in the title field or alternatively "wolff-parkinson-white" in the title, abstract, or keyword fields. The above exclusion criteria were added when it became clear that, without them, a number of papers which are primarily about ALS and Wolff-Parkinson-White syndrome would lessen the accuracy of the analysis through the introduction of data points which are false-positive in nature. The second filter is temporal: only papers 
published and subsequently indexed in WoS between 1 January 1985 and 17 February 2011 were considered. We selected this time period because it is one which has seen remarkable progress and yet is still recent enough that almost all of the investigators in the rankings are still alive. It is important to recognize the effects of the temporal filter as researchers who made fundamental contributions to the field prior to 1985 but have since slowed in the areas of impact and productivity will be underrepresented in this study due to the 1985 limitation. Melvin Yahr and Margaret Hoehn, who, in a landmark paper in 1967, introduced what has long been the standard classification system of PD disease progression, are a case in point. Also of importance is the PD-specific filter, as some prominent PD scientists have strong interests in other areas. Their impact and productivity in non-PD fields will not be recognized in this analysis. John Trojanowski, of the University of Pennsylvania, and Maria Grazia Spillantini, of the University of Cambridge, are prime examples of this phenomenon as their research focuses on the mechanisms which underlie a wide range of neurodegenerative disorders.

Continuing along this line of thought, it seems that analyzing only papers with PD in the title may favor articles on translational or clinical studies for the reason that basic scientists studying the mechanisms underlying PD appear to be less apt to include PD in the titles of their papers. As an example, Spillantini and Trojanowski together with collaborators, Marie Luise Schmidt, Virginia M.-Y. Lee, Ross Jakes, and Michel Goedert, wrote a landmark paper published in Nature in 1997 which mentioned PD in the abstract, but not in the title [5]. Entitled, "alpha-synuclein in Lewy bodies", the paper has accumulated 1,945 citations to date which would have made it the third most-cited paper in the current analysis, had it been included. An analysis of the "false positive" papers which would have been included in the study by relaxing the requirement of a PD-specific title, however, led us to the decision to mandate that PD appear in the title in line with previous studies [1]. A first example of a highly-cited paper which might have been included through the employment of a more relaxed search strategy is "The Consortium to Establish a Registry for Alzheimer's Disease (CERAD). Part II. Standardization of the neuropathologic assessment of Alzheimer's disease" [6], which appeared in Neurology in 1991 and has accumulated 2,444 citations to date. This high-impact AD paper happened to contain PD in its keyword list and is, therefore, returned when one uses the default "Topic" search in WoS. A second example is "Cloning of the gene for a human dopamine D4 receptor with high affinity for the antipsychotic clozapine" [7], which appeared in Nature in 1991 and has accumulated 1,708 citations to date. PD is mentioned in the abstract of this paper, and while the dopamine-receptor research described in this paper may have had a sizeable indirect impact in the world of PD research, it is clear that a paper such as this has its "conceptual home" squarely within the world of schizophrenia investigation.

It is interesting to note that the clinical/translational bias observed in the current analysis appears, anecdotally, to be more apparent than that observed in a similar study conducted in the area of Alzheimer's Disease (AD) [8]. When compared to PD, the historic lack of clinical treatment options for people with AD might, in part, explain why the clinical/translational bias is more evident in the PD study. As demonstrated through the example of the alpha-synuclein paper above, another contributing factor could be the stricter requirement in the current study regarding the appearance of the disease in question in the title of the article.

Reviews were included in the analysis because, while it is relatively "easy" to write a review, it is very difficult to write a review that is well-cited. Well-cited reviews tend to be authored by leaders in the field and present conceptual advances or new hypotheses that can be as important as experimental advances.

While the underlying paper-level data were provided by WoS extracts, the author-specific tabulations of number of papers, "broad impact" citations (see below for further discussion), total citations, and $\mathrm{H}$ index were achieved through the use of the Thomson Reuters HistCite software package [9]. HistCite facilitates author-level bibliometric analysis within a given literature base.

Full names for the PD researchers in this study were derived from the publicly-available BiomedExperts [10] repository, which allows for the accurate extraction of full names (i.e., last name, first name, middle initial) through author-disambiguation algorithms. While powerful, it was clear during the analysis that the automated, author-disambiguation routines used in assigning papers to the individual scientists are not perfect, so manual checking was used in addition. The metrics, therefore, represent a good approximation of impact and productivity rather than an exact measurement.

BiomedExperts.com was also utilized in determining each scientist's main line of investigation. The top five MeSH terms from each investigator's research profile in BiomedExperts were considered. The highest-ranked MeSH term within the top five 
Table 1

Most-cited authors from 1 January 1985 through 17 February 2011 based on citation counts for PD papers indexed in Thomson Reuters ISI WoS and aggregated in Thomson Reuters HistCite software package (institutional affiliation and total number of PD papers in WoS are also indicated)

\begin{tabular}{|c|c|c|c|c|}
\hline $\begin{array}{l}\text { Rank for } \\
\text { Total } \\
\text { Citations }\end{array}$ & $\begin{array}{l}\text { Investigator } \\
(*=\text { Appeared in } \\
\text { JAD Top } 100 \text { analysis [8]) }\end{array}$ & Affiliation & $\begin{array}{c}\text { Total } \\
\text { citations }\end{array}$ & $\begin{array}{c}\text { \# of } \\
\text { PD } \\
\text { Papers }\end{array}$ \\
\hline 1 & Lees, Andrew J & University College London & 23,095 & 459 \\
\hline 2 & Marsden, C David & University College London & 22,235 & 235 \\
\hline 3 & Agid, Yves & Pitié - Salpêtrière University Hospital & 19,699 & 365 \\
\hline 4 & Lang, Anthony E & Toronto Western Research Institute & 16,489 & 365 \\
\hline 5 & Olanow, C Warren & Mount Sinai School of Medicine & 13,759 & 247 \\
\hline 6 & Brooks, David J & Imperial College London & 12,052 & 259 \\
\hline 7 & Jenner, Peter & King's College London & 12,045 & 160 \\
\hline 8 & Mizuno, Yoshikuni & Juntendo University School of Medicine & 10,418 & 324 \\
\hline 9 & Fahn, Stanley & Columbia University & 9,549 & 204 \\
\hline 10 & Benabid, Alim-Louis & Joseph Fourier University & 9,316 & 154 \\
\hline 11 & Goetz, Christopher G & Rush University Medical Center & 9,191 & 318 \\
\hline 12 & Quinn, Niall P & University College London & 9,184 & 228 \\
\hline 13 & Pollak, Pierre & Joseph Fourier University & 9,008 & 196 \\
\hline 14 & Hirsch, Etienne C & Pitié - Salpêtrière University Hospital & 8,891 & 177 \\
\hline 15 & Koller, William C & University of North Carolina & 8,853 & 255 \\
\hline 16 & Lozano, Andres M & University of Toronto & 8,349 & 165 \\
\hline 17 & Riederer, Peter* & Universität Würzburg & 8,242 & 159 \\
\hline 18 & Jankovic, Joseph & Baylor College of Medicine & 8,023 & 318 \\
\hline 19 & Daniel, Susan E & University College London & 7,284 & 211 \\
\hline 19 & Tanner, Caroline M & The Parkinson's Institute & 7,284 & 55 \\
\hline 21 & Farrer, Matthew J & Mayo Clinic - Florida & 7,241 & 237 \\
\hline 22 & Obeso, Jose A & University of Navarra & 7,173 & 201 \\
\hline 23 & Björklund, Anders & Lund University & 7,040 & 90 \\
\hline 24 & Hattori, Nobutaka & Juntendo University School of Medicine & 6,864 & 228 \\
\hline 25 & Youdim, Moussa B H & Technion - Israel Institute of Technology & 6,667 & 133 \\
\hline 26 & Langston, J William & The Parkinson's Institute & 6,661 & 149 \\
\hline 27 & Golbe, Lawrence I & University of Medicine and Dentistry of New Jersey & 6,561 & 84 \\
\hline 28 & Schapira, Anthony H V & University College London & 6,515 & 170 \\
\hline 29 & Przedborski, Serge & Columbia University & 6,512 & 83 \\
\hline 30 & Calne, Donald B & University of British Columbia & 6,393 & 156 \\
\hline 31 & Nutt, John G & Oregon Health \& Science University & 6,320 & 220 \\
\hline 32 & Rascol, Olivier & University of Toulouse & 6,144 & 340 \\
\hline 33 & Wood, Nicholas W & University College London & 6,021 & 144 \\
\hline 34 & Gasser, Thomas & University of Tübingen & 5,926 & 160 \\
\hline 35 & Poewe, Werner & Innsbruck Medical University & 5,892 & 326 \\
\hline 36 & Dexter, David T & Imperial College London & 5,846 & 45 \\
\hline 37 & Jellinger, Kurt A* & University of Vienna & 5,797 & 116 \\
\hline 38 & Nussbaum, Robert L & University of California San Francisco & 5,770 & 32 \\
\hline 39 & Javoy-Agid, France & Pitié - Salpêtrière University Hospital & 5,697 & 78 \\
\hline 40 & Chase, Thomas N & Hamilton Pharmaceuticals. & 5,668 & 137 \\
\hline 41 & Maraganore, Demetrius M & Mayo Clinic - Minnesota & 5,633 & 153 \\
\hline 42 & Bonifati, Vincenzo & Erasmus University & 5,611 & 136 \\
\hline 43 & DeLong, Mahlon R & Emory University & 5,394 & 73 \\
\hline 44 & Shoulson, Ira & University of Rochester & 5,371 & 87 \\
\hline 45 & Oertel, Wolfgang $\mathrm{H}$ & The Philipps University & 5,317 & 279 \\
\hline 46 & Stern, Matthew B & University of Pennsylvania & 5,284 & 212 \\
\hline 47 & Duvoisin, Roger C & University of Medicine and Dentistry of New Jersey & 5,193 & 54 \\
\hline 48 & Krack, Paul & Joseph Fourier University & 5,143 & 101 \\
\hline 49 & Bonnet, Anne-Marie & Pitié - Salpêtrière University Hospital & 5,131 & 116 \\
\hline 50 & Singleton, Andrew & National Institute of Aging - NIH & 5,108 & 117 \\
\hline 51 & Larsen, Jan Petter & Stavanger University Hospital & 5,053 & 170 \\
\hline 52 & Hughes, Andrew J & University of Melbourne & 5,017 & 47 \\
\hline 53 & Kieburtz, Karl & University of Rochester & 5,010 & 92 \\
\hline 54 & Mayeux, Richard* & Columbia University & 4,919 & 103 \\
\hline 55 & Rothwell, John C & University College London & 4,825 & 85 \\
\hline 56 & Hardy, John* & University College London & 4,818 & 131 \\
\hline 57 & Brice, Alexis & Pitié - Salpêtrière University Hospital & 4,802 & 135 \\
\hline
\end{tabular}


Table 1 (continued)

\begin{tabular}{|c|c|c|c|c|}
\hline $\begin{array}{l}\text { Rank for } \\
\text { Total } \\
\text { Citations }\end{array}$ & $\begin{array}{l}\text { Investigator } \\
(*=\text { Appeared in } \\
\text { JAD Top } 100 \text { analysis [8] })\end{array}$ & Affiliation & $\begin{array}{c}\text { Total } \\
\text { citations }\end{array}$ & $\begin{array}{c}\text { \# of } \\
\text { PD } \\
\text { Papers }\end{array}$ \\
\hline 58 & Limousin, Patricia & University College London & 4,752 & 73 \\
\hline 59 & Brundin, Patrik & Lund University & 4,731 & 65 \\
\hline 60 & Eidelberg, David & Feinstein Institute for Medical Research & 4,683 & 203 \\
\hline 61 & Albanese, Alberto & Università Cattolica del Sacro Cuore & 4,674 & 92 \\
\hline 62 & Pahwa, Rajesh & University of Kansas & 4,636 & 193 \\
\hline 63 & LeWitt, Peter & Wayne State University & 4,553 & 113 \\
\hline 64 & Polymeropoulos, Mihael H & Vanda Pharmaceuticals & 4,531 & 20 \\
\hline 65 & Dawson, Ted M & Johns Hopkins University & 4,518 & 77 \\
\hline 66 & Lindvall, Olle & Lund University & 4,514 & 65 \\
\hline 67 & Robbins, Trevor W & University of Cambridge & 4,510 & 48 \\
\hline 68 & Frackowiak, Richard S J & University College London & 4,507 & 29 \\
\hline 69 & Aarsland, Dag & Stavanger University Hospital & 4,476 & 167 \\
\hline 70 & Hauser, Robert A & University of South Florida & 4,451 & 181 \\
\hline 71 & Rajput, Ali H & University of Saskatchewan & 4,369 & 126 \\
\hline 72 & Pfeiffer, Ronald F & University of Tennessee & 4,307 & 114 \\
\hline 73 & Wszolek, Zbigniew K & Mayo Clinic - Florida & 4,232 & 179 \\
\hline 74 & Benazzouz, Abdelhamid & Université de Bordeaux & 4,212 & 37 \\
\hline 75 & Kordower, Jeffrey & Rush University Medical Center & 4,203 & 90 \\
\hline 76 & Marek, Kenneth & Institute for Neurodegenerative Disorders & 4,176 & 136 \\
\hline 77 & Weiner, William J & University of Maryland - Baltimore & 4,146 & 172 \\
\hline 78 & Rehncrona, Stig & Lund University & 4,069 & 224 \\
\hline 78 & Tolosa, Eduardo & University of Barcelona & 4,069 & 40 \\
\hline 80 & Hubble, Jean P & Novartis Pharmaceuticals Corporation & 4,057 & 90 \\
\hline 81 & Watts, Ray L & University of Alabama at Birmingham & 4,053 & 151 \\
\hline 82 & Trojanowski, John $\mathrm{Q}^{*}$ & University of Pennsylvania & 4,019 & 50 \\
\hline 83 & Jackson-Lewis, Vernice & Columbia University & 3,995 & 48 \\
\hline 84 & Sawle, Guy V & Nottingham University & 3,989 & 34 \\
\hline 85 & Müller, Thomas & St. Joseph Hospital Berlin-Weissensee & 3,957 & 163 \\
\hline 86 & Widner, Håkan & Lund University & 3,933 & 60 \\
\hline 87 & Stoessl, A Jon & University of British Columbia & 3,917 & 109 \\
\hline 88 & Meco, Giuseppe & Sapienza University & 3,905 & 96 \\
\hline 89 & Shults, Clifford W & University of California San Diego & 3,827 & 64 \\
\hline 90 & Beal, M Flint* & Cornell University & 3,776 & 61 \\
\hline 91 & Leenders, Klaus L & University Medical Center Groningen & 3,771 & 108 \\
\hline 92 & Vila, Miquel & University Hospital Vall d'Hebron & 3,754 & 43 \\
\hline 93 & Greenamyre, J Timothy & University of Pittsburgh & 3,751 & 53 \\
\hline 94 & Johnson, William G & University of Medicine and Dentistry of New Jersey & 3,686 & 13 \\
\hline 95 & Lazzarini, Alice M & University of Medicine and Dentistry of New Jersey & 3,682 & 13 \\
\hline 96 & Friedman, Joseph H & Brown University & 3,671 & 165 \\
\hline 97 & Dawson, Valina L & Johns Hopkins University & 3,646 & 45 \\
\hline 98 & Gwinn-Hardy, Katrina & National Institute of Neurological Disorders and Stroke - NIH & 3,629 & 77 \\
\hline 99 & Przuntek, Horst & Ruhr-University of Bochum & 3,583 & 107 \\
\hline 100 & Lansbury, Peter T & Harvard University & 3,557 & 28 \\
\hline
\end{tabular}

for a given scientist that could be considered a PD line of investigation was chosen as the main line of investigation for the researcher in question. It is important to note that the papers used to generate the top five MeSH terms for each scientist were not restricted to those papers mentioning PD, but were taken from a collection of PubMed papers representative of an investigator's entire corpus of published work. It follows, then, that the line of investigation chosen for each researcher is not necessarily the line of investigation most frequently found within that scientist's PD papers. The MeSH term chosen is, however, one that the scientist has applied to their PD research and, more importantly, is the line of investigation most representative of the entire research portfolio of the investigator in question. This approach to line-of-investigation identification, while not always completely accurate, allows a broad picture of the areas of expertise of the most cited researchers within the world of PD research without attempting to compare competing $\mathrm{MeSH}$-term frequencies within an investigator's PD-specific paper corpus. 
Table 2

Authors with the most broad-impact citations using the same document base as Table 1 (institutional affiliation and total citations are also indicated)

\begin{tabular}{|c|c|c|c|c|}
\hline $\begin{array}{l}\text { Rank for Broad } \\
\text { impact citations }\end{array}$ & Investigator & Affiliation & $\begin{array}{c}\text { Broad impact } \\
\text { citations }\end{array}$ & $\begin{array}{c}\text { Total } \\
\text { citations }\end{array}$ \\
\hline 1 & Marsden, C David & University College London & 10,783 & 22,235 \\
\hline 2 & Agid, Yves & Pitié - Salpêtrière University Hospital & 9,745 & 19,699 \\
\hline 3 & Lees, Andrew J & University College London & 8,452 & 23,095 \\
\hline 4 & Jenner, Peter & King's College London & 7,547 & 12,045 \\
\hline 5 & Olanow, C Warren & Mount Sinai School of Medicine & 7,440 & 13,759 \\
\hline 6 & Lang, Anthony E & Toronto Western Research Institute & 5,979 & 16,489 \\
\hline 7 & Hirsch, Etienne C & Pitié - Salpêtrière University Hospital & 5,473 & 8,891 \\
\hline 8 & Mizuno, Yoshikuni & Juntendo University School of Medicine & 5,296 & 10,418 \\
\hline 9 & Brooks, David J & Imperial College London & 5,161 & 12,052 \\
\hline 10 & Riederer, Peter & Universität Würzburg & 5,012 & 8,242 \\
\hline 11 & Youdim, Moussa B H & Technion - Israel Institute of Technology & 4,322 & 6,667 \\
\hline 12 & Benabid, Alim-Louis & Joseph Fourier University & 4,234 & 9,316 \\
\hline 13 & Björklund, Anders & Lund University & 4,077 & 7,040 \\
\hline 14 & Przedborski, Serge & Columbia University & 3,886 & 6,512 \\
\hline 15 & Pollak, Pierre & University of Grenoble & 3,727 & 9,008 \\
\hline 16 & Fahn, Stanley & Columbia University & 3,676 & 9,549 \\
\hline 17 & Lozano, Andres M & University of Toronto & 3,675 & 8,349 \\
\hline 18 & Koller, William C & University of North Carolina & 3,573 & 8,853 \\
\hline 19 & Quinn, Niall P & University College London & 3,536 & 9,184 \\
\hline 20 & Obeso, Jose A & University of Navarra & 3,487 & 7,173 \\
\hline 21 & Schapira, Anthony H V & University College London & 3,443 & 6,515 \\
\hline 22 & Hattori, Nobutaka & Juntendo University School of Medicine & 3,405 & 6,864 \\
\hline 23 & Dexter, David T & Imperial College London & 3,386 & 5,846 \\
\hline 24 & Javoy-Agid, France & Pitié - Salpêtrière University Hospital & 3,368 & 5,697 \\
\hline 25 & Jellinger, Kurt A & University of Vienna & 3,268 & 5,797 \\
\hline 26 & Singleton, Andrew & National Institute of Aging - NIH & 3,222 & 5,108 \\
\hline 27 & Langston, J William & The Parkinson's Institute & 3,180 & 6,661 \\
\hline 28 & Jankovic, Joseph & Baylor College of Medicine & 3,134 & 8,023 \\
\hline 29 & Goetz, Christopher G & Rush University Medical Center & 3,081 & 9,191 \\
\hline 30 & Nussbaum, Robert L & University of California San Francisco & 2,983 & 5,770 \\
\hline 31 & Calne, Donald B & University of British Columbia & 2,776 & 6,393 \\
\hline 32 & Sawle, Guy V & Nottingham University & 2,769 & 3,989 \\
\hline 33 & Beal, M Flint & Cornell University & 2,757 & 3,776 \\
\hline 34 & Brundin, Patrik & Lund University & 2,752 & 4,731 \\
\hline 35 & Trojanowski, John Q & University of Pennsylvania & 2,725 & 4,019 \\
\hline 36 & Farrer, Matthew J & Mayo Clinic - Florida & 2,689 & 7,241 \\
\hline 37 & Daniel, Susan E & University College London & 2,674 & 7,284 \\
\hline 38 & Rajput, Ali H & University of Saskatchewan & 2,658 & 4,369 \\
\hline 39 & Lansbury, Peter T & Harvard University & 2,655 & 3,557 \\
\hline 40 & DeLong, Mahlon R & Emory University & 2,649 & 5,394 \\
\hline 41 & Limousin, Patricia & University College London & 2,630 & 4,752 \\
\hline 42 & Lee, Virginia M-Y & University of Pennsylvania & 2,576 & 3,544 \\
\hline 43 & Dawson, Ted M & Johns Hopkins University & 2,575 & 4,518 \\
\hline 44 & Golbe, Lawrence I & University of Medicine and Dentistry of New Jersey & 2,551 & 6,561 \\
\hline 45 & Lindvall, Olle & Lund University & 2,540 & 4,514 \\
\hline 46 & Jackson-Lewis, Vernice & Columbia University & 2,529 & 3,995 \\
\hline 47 & Robbins, Trevor W & University of Cambridge & 2,476 & 4,510 \\
\hline 48 & Chase, Thomas N & Hamilton Pharmaceuticals. & 2,458 & 5,668 \\
\hline 49 & Vila, Miquel & University Hospital Vall d'Hebron & 2,398 & 3,754 \\
\hline 50 & Greenamyre, J Timothy & University of Pittsburgh & 2,391 & 3,751 \\
\hline 51 & Kordower, Jeffrey & Rush University Medical Center & 2,386 & 4,203 \\
\hline 52 & Nutt, John G & Oregon Health \& Science University & 2,300 & 6,320 \\
\hline 53 & Tanner, Caroline M & The Parkinson's Institute & 2,296 & 7,284 \\
\hline 54 & Polymeropoulos, Mihael H & Vanda Pharmaceuticals & 2,268 & 4,531 \\
\hline 55 & Rothwell, John C & University College London & 2,243 & 4,825 \\
\hline 56 & Frackowiak, Richard S J & University College London & 2,186 & 4,507 \\
\hline 57 & Dawson, Valina L & Johns Hopkins University & 2,170 & 3,646 \\
\hline 58 & Krack, Paul & University of Grenoble & 2,136 & 5,143 \\
\hline 59 & Gasser, Thomas & University of Tübingen & 2,121 & 5,926 \\
\hline
\end{tabular}


Table 2 (continued)

\begin{tabular}{|c|c|c|c|c|}
\hline $\begin{array}{l}\text { Rank for Broad } \\
\text { impact citations }\end{array}$ & Investigator & Affiliation & $\begin{array}{c}\text { Broad impact } \\
\text { citations }\end{array}$ & $\begin{array}{c}\text { Total } \\
\text { citations }\end{array}$ \\
\hline 60 & Oertel, Wolfgang $\mathrm{H}$ & The Philipps University & 2,104 & 5,317 \\
\hline 61 & Eidelberg, David & Feinstein Institute for Medical Research & 2,102 & 4,683 \\
\hline 62 & Widner, Håkan & Lund University & 2,100 & 3,933 \\
\hline 63 & Wood, Nicholas W & University College London & 2,090 & 6,021 \\
\hline 64 & Duvoisin, Roger C & University of Medicine and Dentistry of New Jersey & 2,087 & 5,193 \\
\hline 65 & Poewe, Werner & Innsbruck Medical University & 2,062 & 5,892 \\
\hline 66 & Rascol, Olivier & University of Toulouse & 2,043 & 6,144 \\
\hline 67 & Mayeux, Richard & Columbia University & 2,036 & 4,919 \\
\hline 68 & Rehncrona, Stig & Lund University & 2,035 & 4,069 \\
\hline 69 & Crossman, Alan R & University of Manchester & 2,012 & 3,523 \\
\hline 70 & Maraganore, Demetrius M & Mayo Clinic - Minnesota & 1,968 & 5,633 \\
\hline 71 & Watts, Ray L & University of Alabama at Birmingham & 1,965 & 4,053 \\
\hline 72 & Müller, Thomas & St. Joseph Hospital Berlin-Weissensee & 1,959 & 3,957 \\
\hline 73 & Stern, Matthew B & University of Pennsylvania & 1,953 & 5,284 \\
\hline 74 & Hardy, John & University College London & 1,932 & 4,818 \\
\hline 75 & Perry, Robert H & Newcastle University & 1,892 & 2,729 \\
\hline 76 & Bonnet, Anne-Marie & Pitié - Salpêtrière University Hospital & 1,872 & 5,131 \\
\hline 77 & Leenders, Klaus L & University Medical Center Groningen & 1,861 & 3,771 \\
\hline 78 & Nagatsu, Toshiharu & Fujita Health University & 1,860 & 3,104 \\
\hline 79 & Benazzouz, Abdelhamid & Université de Bordeaux & 1,847 & 4,212 \\
\hline 80 & Brice, Alexis & Pitié - Salpêtrière University Hospital & 1,826 & 4,802 \\
\hline 81 & Stoessl, A Jon & University of British Columbia & 1,784 & 3,917 \\
\hline 82 & Przuntek, Horst & Ruhr-University of Bochum & 1,778 & 3,583 \\
\hline 83 & Bonifati, Vincenzo & Erasmus University & 1,772 & 5,611 \\
\hline 84 & Spillantini, Maria Grazia & University of Cambridge & 1,757 & 2,313 \\
\hline 85 & Hauser, Robert A & University of South Florida & 1,747 & 4,451 \\
\hline 86 & Johnson, William G & University of Medicine and Dentistry of New Jersey & 1,735 & 3,686 \\
\hline 87 & Lazzarini, Alice M & University of Medicine and Dentistry of New Jersey & 1,724 & 3,682 \\
\hline 88 & Albanese, Alberto & Università Cattolica del Sacro Cuore & 1,703 & 4,674 \\
\hline 89 & Pahwa, Rajesh & University of Kansas & 1,698 & 4,636 \\
\hline 90 & Minoshima, Satoshi & University of Washington & 1,649 & 3,215 \\
\hline 91 & Cooper, J Mark & University College London & 1,639 & 2,985 \\
\hline 92 & Wszolek, Zbigniew K & Mayo Clinic - Florida & 1,633 & 4,232 \\
\hline 93 & Shoulson, Ira & University of Rochester & 1,626 & 5,371 \\
\hline 94 & Shults, Clifford W & University of California San Diego & 1,624 & 3,827 \\
\hline 95 & Goedert, Michel & University of Cambridge & 1,620 & 2,226 \\
\hline 96 & LeWitt, Peter & Wayne State University & 1,597 & 4,553 \\
\hline 97 & McGeer, Edith G & University of British Columbia & 1,585 & 2,460 \\
\hline 98 & Kieburtz, Karl & University of Rochester & 1,582 & 5,010 \\
\hline 98 & Cummings, Jeffrey L & University of California, Los Angeles & 1,582 & 2,962 \\
\hline 100 & McGeer, Patrick L & University of British Columbia & 1,575 & 2,456 \\
\hline
\end{tabular}

As part of the total-citations analysis, the new metric, "broad impact citations", is used as a measure of a given investigator's impact beyond the PD research community. In the broad-impact ranking, only the subset of an investigator's citations which originate from references in papers that are not part of this analysis (i.e. papers which fall outside of the PD literature) are considered.

For the 21 st century ranking, the same methods and considerations were applied to the data collection and analysis as described for the post-1984 total-citations ranking. The only difference is the temporal filter applied, which, for the 21 st century ranking, was PD papers published and indexed in WoS between 2001 and 2010.

\section{RESULTS}

\section{Productivity and impact among PD investigators - the three metrics}

As a first step in determining which PD investigators have contributed the most to the field since 1985, an article corpus was generated comprising 40,152 papers written in 21 languages appearing in 2,387 journals by authors representing 108 countries. From this corpus, a pool of the 300 most-cited PD researchers was generated. Those investigators who had five or fewer PD papers were excluded from the analysis. For each author in the pool of the 300 most-cited investigators, $\mathrm{H}$-indices, total paper counts, "broad impact" citations 
Table 3

Authors with highest $\mathrm{H}$-indices calculated from same document base as used for the total-citation tabulations in Table 1 (total number of PD papers in WoS and the main line of investigation from BiomedExperts for each scientist are also indicated)

\begin{tabular}{|c|c|c|c|c|}
\hline $\begin{array}{l}\text { PD H-Index } \\
\text { Rank } \\
\end{array}$ & $\begin{array}{l}\text { Investigator }(*=\text { Appeared } \\
\text { in JAD Top } 100 \text { analysis [8]) }\end{array}$ & Main line of investigation & $\begin{array}{c}\text { PD } \\
\text { H-Index }\end{array}$ & $\begin{array}{r}\text { \# of PD } \\
\text { Papers }\end{array}$ \\
\hline 1 & Agid, Yves & Dopamine & 77 & 365 \\
\hline 1 & Marsden, C David & Dopamine & 77 & 235 \\
\hline 3 & Lees, Andrew J & Levodopa & 73 & 459 \\
\hline 4 & Lang, Anthony E & Levodopa & 65 & 365 \\
\hline 5 & Olanow, C Warren & Levodopa & 58 & 247 \\
\hline 6 & Brooks, David J & Emission-Computed Tomography & 57 & 259 \\
\hline 7 & Goetz, Christopher G & Levodopa & 56 & 318 \\
\hline 8 & Quinn, Niall P & Levodopa & 54 & 228 \\
\hline 9 & Hirsch, Etienne C & Dopamine & 52 & 177 \\
\hline 10 & Koller, William C & Levodopa & 51 & 255 \\
\hline 11 & Jenner, Peter & Dopamine & 50 & 160 \\
\hline 12 & Fahn, Stanley & Levodopa & 49 & 204 \\
\hline 12 & Jankovic, Joseph & Botulinum Toxins & 49 & 318 \\
\hline 14 & Obeso, Jose A & Levodopa & 48 & 201 \\
\hline 14 & Pollak, Pierre & Electric Stimulation Therapy & 48 & 196 \\
\hline 14 & Tanner, Caroline $\mathrm{M}$ & Levodopa & 48 & 211 \\
\hline 17 & Riederer, Peter* & Dopamine & 46 & 159 \\
\hline 18 & Rascol, Olivier & Levodopa & 45 & 340 \\
\hline 19 & Benabid, Alim-Louis & Electric Stimulation Therapy & 44 & 154 \\
\hline 19 & Björklund, Anders & Dopamine & 44 & 90 \\
\hline 19 & Oertel, Wolfgang $\mathrm{H}$ & Levodopa & 44 & 279 \\
\hline 19 & Przedborski, Serge & 1-Methyl-4-phenyl-1,2,3,6-tetrahydropyridine & 44 & 83 \\
\hline 23 & Mizuno, Yoshikuni & Ubiquitin-Protein Ligases & 43 & 324 \\
\hline 23 & Nutt, John G & Levodopa & 43 & 220 \\
\hline 23 & Poewe, Werner & Levodopa & 43 & 326 \\
\hline 23 & Youdim, Moussa B H & Monoamine Oxidase & 43 & 133 \\
\hline 27 & Chase, Thomas N & Levodopa & 42 & 137 \\
\hline 27 & Farrer, Matthew J & Genetic Predisposition to Disease & 42 & 237 \\
\hline 27 & Larsen, Jan Petter & Neuropsychological Tests & 42 & 170 \\
\hline 27 & Lozano, Andres M & Deep Brain Stimulation & 42 & 165 \\
\hline 31 & Calne, Donald B & Levodopa & 40 & 156 \\
\hline 31 & Langston, J William & 1-Methyl-4-phenyl-1,2,3,6-tetrahydropyridine & 40 & 149 \\
\hline 33 & Bonifati, Vincenzo & Ubiquitin-Protein Ligases & 39 & 136 \\
\hline 33 & Jellinger, Kurt A* & Autopsy & 39 & 116 \\
\hline 35 & Aarsland, Dag & Neuropsychological Tests & 38 & 167 \\
\hline 35 & Mayeux, Richard* & Apolipoproteins E & 38 & 103 \\
\hline 35 & Schapira, Anthony H V & Mitochondrial DNA & 38 & 170 \\
\hline 35 & Stern, Matthew B & Levodopa & 38 & 212 \\
\hline 39 & Bonnet, Anne-Marie & Levodopa & 37 & 116 \\
\hline 39 & Maraganore, Demetrius M & Genetic Predisposition to Disease & 37 & 153 \\
\hline 41 & Eidelberg, David & Brain Mapping & 36 & 203 \\
\hline 41 & Javoy-Agid, France & Dopamine & 36 & 78 \\
\hline 43 & Ahlskog, J Eric & Levodopa & 35 & 145 \\
\hline 43 & Côté, Lucien J & Neuropsychological Tests & 35 & 106 \\
\hline 43 & Friedman, Joseph H & Clozapine & 35 & 165 \\
\hline 43 & Gasser, Thomas & DNA Mutational Analysis & 35 & 160 \\
\hline 43 & LeWitt, Peter & Levodopa & 35 & 113 \\
\hline 43 & Marder, Karen & Neuropsychological Tests & 35 & 128 \\
\hline 43 & Rajput, Ali H & Levodopa & 35 & 126 \\
\hline 50 & Crossman, Alan R & Levodopa & 34 & 92 \\
\hline 50 & Golbe, Lawrence I & alpha-synuclein & 34 & 84 \\
\hline 50 & Hubble, Jean P & Levodopa & 34 & 90 \\
\hline 50 & Rinne, Juha O & Emission Computed Tomography & 34 & 90 \\
\hline 54 & Daniel, Susan E & Lewy Bodies & 33 & 55 \\
\hline 54 & Hardy, John* & Genetic Predisposition to Disease/tau Proteins & 33 & 131 \\
\hline 54 & Hattori, Nobutaka & Ubiquitin-Protein Ligases & 33 & 228 \\
\hline 54 & Krack, Paul & Electric Stimulation Therapy & 33 & 101 \\
\hline 54 & Montastruc, Jean-Louis & Levodopa & 33 & 127 \\
\hline 54 & Pahwa, Rajesh & Deep Brain Stimulation & 33 & 193 \\
\hline
\end{tabular}


Table 3 (continued)

\begin{tabular}{|c|c|c|c|c|}
\hline $\begin{array}{l}\text { PD H-Index } \\
\text { Rank }\end{array}$ & $\begin{array}{l}\text { Investigator }(*=\text { Appeared } \\
\text { in JAD Top } 100 \text { analysis [8]) }\end{array}$ & Main line of investigation & $\begin{array}{c}\text { PD } \\
\text { H-Index }\end{array}$ & $\begin{array}{c}\text { \# of PD } \\
\text { Papers }\end{array}$ \\
\hline 54 & Pfeiffer, Ronald F & Levodopa & 33 & 114 \\
\hline 54 & Rinne, Urpo K & Levodopa & 33 & 101 \\
\hline 54 & Tolosa, Eduardo & Levodopa & 33 & 224 \\
\hline 63 & Brice, Alexis & Pedigree (genealogy) & 32 & 135 \\
\hline 63 & Melamed, Eldad & Dopamine & 32 & 154 \\
\hline 63 & Watts, Ray L & Genetic Predisposition to Disease & 32 & 151 \\
\hline 63 & Weiner, William J & Levodopa & 32 & 172 \\
\hline 67 & Burn, David J & Differential Diagnosis & 31 & 160 \\
\hline 67 & Factor, Stewart A & Levodopa & 31 & 117 \\
\hline 67 & Singleton, Andrew & Genetic Predisposition to Disease & 31 & 117 \\
\hline 67 & Stocchi, Fabrizio & Lisuride & 31 & 179 \\
\hline 67 & Wood, Nicholas W & Pedigree (genealogy) & 31 & 144 \\
\hline 67 & Wszolek, Zbigniew K & Pedigree (genealogy) & 31 & 179 \\
\hline 73 & Brotchie, Jonathan M & Levodopa & 30 & 136 \\
\hline 73 & Dawson, Ted M & Nitric Oxide Synthase & 30 & 77 \\
\hline 73 & DeLong, Mahlon R & Brain Mapping & 30 & 73 \\
\hline 73 & Giladi, Nir & Levodopa & 30 & 245 \\
\hline 73 & Hauser, Robert A & Levodopa & 30 & 181 \\
\hline 73 & Klein, Christine & DNA Mutational Analysis & 30 & 171 \\
\hline 73 & McKeith, Ian G* & Lewy Bodies & 30 & 81 \\
\hline 73 & Meco, Giuseppe & Levodopa & 30 & 96 \\
\hline 73 & Wenning, Gregor & Differential Diagnosis & 30 & 105 \\
\hline 82 & Beal, M Flint* & Mitochondria & 29 & 61 \\
\hline 82 & Deuschl, Günther & Deep Brain Stimulation & 29 & 140 \\
\hline 82 & Dhawan, Vijay & Emission-Computed Tomography & 29 & 126 \\
\hline 82 & Dubois, Bruno & Neuropsychological Tests & 29 & 77 \\
\hline 82 & Jackson-Lewis, Vernice & Dopamine & 29 & 48 \\
\hline 82 & Kieburtz, Karl & Neuropsychological Tests & 29 & 92 \\
\hline 82 & Korczyn, Amos D & Risk Factors & 29 & 127 \\
\hline 82 & Nagatsu, Toshiharu & Tyrosine 3-Monooxygenase & 29 & 81 \\
\hline 82 & Rocca, Walter A & Risk Factors & 29 & 126 \\
\hline 82 & Rothwell, John C & Transcranial Magnetic Stimulation & 29 & 85 \\
\hline 82 & Vieregge, Peter & Pedigree (genealogy) & 29 & 96 \\
\hline 82 & Wolters, Erik C & Dopamine & 29 & 138 \\
\hline 94 & Albanese, Alberto & Botulinum Toxins & 28 & 92 \\
\hline 94 & Brundin, Patrik & Dopamine & 28 & 65 \\
\hline 94 & Gwinn-Hardy, Katrina & Synucleins & 28 & 77 \\
\hline 94 & Hurtig, Howard I & Levodopa & 28 & 103 \\
\hline 94 & Kurlan, Roger & Levodopa & 28 & 65 \\
\hline 94 & Lindvall, Olle & Brain Tissue Transplantation & 28 & 65 \\
\hline 94 & Marek, Kenneth & Dopamine Plasma Membrane Transport Proteins & 28 & 136 \\
\hline 94 & Perry, Robert H* & Lewy Bodies & 28 & 60 \\
\hline 94 & Shoulson, Ira & Levodopa & 28 & 87 \\
\hline 94 & Stebbins, Glenn T & Severity of Illness Index & 28 & 106 \\
\hline
\end{tabular}

(see the section below), and total citations were calculated. Finally top-100 rankings for total citations and $\mathrm{H}$-Index were generated.

\section{Further refining impact measures - broad impact citations}

Because of the increasing phenomenon of advances having implications beyond a particular disease, part of the analysis was dedicated to evaluating methods of differentiating, algorithmically, those authors who contribute work that has impact beyond the PD community. One datum generated by HistCite is the number of "internal" citations an author has generated within a given set of papers. Applying the concept of internal citations to the PD literature under analysis, one is able to split each investigator's citations into those citations accrued from papers within the PD literature and those citations arising from references in papers outside of the PD literature. It thus becomes possible to calculate how many citations a given investigator has if only citations from papers outside the PD literature were 
Table 4

Most-cited authors from 2001 to 2010 based on citation rates for PD papers indexed in Thomson Reuters ISI WoS and aggregated in Thomson Reuters HistCite (institutional affiliation, rank from Table 1, and rise or fall in rank between Tables 1 and 4 indicated). (n/a) indicates new names that appear in the most cited of the last 10 years who did not appear in the 25 year analysis

\begin{tabular}{|c|c|c|c|c|c|}
\hline $\begin{array}{l}\text { Rank for } \\
\text { total } \\
\text { citations }\end{array}$ & Investigator & Affiliation & $\begin{array}{c}\text { Total } \\
\text { citations }\end{array}$ & $\begin{array}{c}\text { Table } 1 \\
\text { total } \\
\text { citations } \\
\text { rank }\end{array}$ & $\begin{array}{c}\text { Rise or fall } \\
\text { in Rank } \\
\text { from Table } 1 \\
\text { to Table } 4\end{array}$ \\
\hline 1 & Lang, Anthony E & Toronto Western Research Institute & 7,464 & 4 & 3 \\
\hline 2 & Farrer, Matthew J & Mayo Clinic - Florida & 6,470 & 21 & 19 \\
\hline 3 & Lees, Andrew J & University College London & 5,999 & 1 & -2 \\
\hline 4 & Olanow, C Warren & Mount Sinai School of Medicine & 5,905 & 5 & 1 \\
\hline 5 & Singleton, Andrew & National Institute of Aging - NIH & 5,007 & 50 & 45 \\
\hline 6 & Przedborski, Serge & Columbia University & 4,477 & 29 & 23 \\
\hline 7 & Wood, Nicholas W & University College London & 4,396 & 33 & 26 \\
\hline 8 & Pollak, Pierre & Joseph Fourier University & 4,311 & 13 & 5 \\
\hline 9 & Benabid, Alim-Louis & Joseph Fourier University & 4,123 & 10 & 1 \\
\hline 10 & Poewe, Werner & Innsbruck Medical University & 4,107 & 35 & 25 \\
\hline 11 & Jankovic, Joseph & Baylor College of Medicine & 4,094 & 18 & 7 \\
\hline 12 & Fahn, Stanley & Columbia University & 3,985 & 9 & -3 \\
\hline 13 & Hardy, John & University College London & 3,964 & 56 & 43 \\
\hline 14 & Agid, Yves & Pitié - Salpêtrière University Hospital & 3,759 & 3 & -11 \\
\hline 15 & Lozano, Andres M & University of Toronto & 3,700 & 16 & 1 \\
\hline 16 & Albanese, Alberto & Università Cattolica del Sacro Cuore & 3,691 & 61 & 45 \\
\hline 17 & Goetz, Christopher G & Rush University Medical Center & 3,690 & 11 & -6 \\
\hline 18 & Dawson, Ted M & Johns Hopkins University & 3,687 & 65 & 47 \\
\hline 19 & Cookson, Mark R & National Institute on Aging - NIH & 3,560 & $\mathrm{n} / \mathrm{a}$ & $\mathrm{n} / \mathrm{a}$ \\
\hline 20 & Maraganore, Demetrius M & Mayo Clinic - Minnesota & 3,504 & 41 & 21 \\
\hline 21 & Aarsland, Dag & Stavanger University Hospital & 3,441 & 69 & 48 \\
\hline 22 & Brooks, David J & Imperial College London & 3,438 & 6 & -16 \\
\hline 23 & Stoessl, A Jon & University of British Columbia & 3,391 & 87 & 64 \\
\hline 24 & Koller, William C & University of North Carolina & 3,313 & 15 & -9 \\
\hline 25 & Kachergus, Jennifer & Mayo Clinic - Florida & 3,300 & $\mathrm{n} / \mathrm{a}$ & $\mathrm{n} / \mathrm{a}$ \\
\hline 26 & Bonifati, Vincenzo & Erasmus University & 3,299 & 42 & 16 \\
\hline 27 & Gasser, Thomas & University of Tübingen & 3,170 & 34 & 7 \\
\hline 28 & Brice, Alexis & Pitié - Salpêtrière University Hospital & 3,136 & 57 & 29 \\
\hline 29 & Tolosa, Eduardo & University of Barcelona & 3,115 & 78 & 49 \\
\hline 30 & Gwinn-Hardy, Katrina & National Institute of Neurological Disorders and Stroke - NIH & 3,073 & 98 & 68 \\
\hline 31 & Quinn, Niall P & University College London & 3,049 & 12 & -19 \\
\hline 32 & Stern, Matthew B & University of Pennsylvania & 3,015 & 46 & 14 \\
\hline 33 & Jackson-Lewis, Vernice & Columbia University & 2,929 & 83 & 50 \\
\hline 34 & Pahwa, Rajesh & University of Kansas & 2,915 & 62 & 28 \\
\hline 35 & Mizuno, Yoshikuni & Juntendo University School of Medicine & 2,892 & 8 & -27 \\
\hline 36 & Obeso, Jose A & University of Navarra & 2,887 & 22 & -14 \\
\hline 37 & Krack, Paul & Joseph Fourier University & 2,874 & 48 & 11 \\
\hline 38 & Dawson, Valina L & Johns Hopkins University & 2,865 & 97 & 59 \\
\hline 39 & Eidelberg, David & Feinstein Institute for Medical Research & 2,816 & 60 & 21 \\
\hline 40 & Kieburtz, Karl & University of Rochester & 2,751 & 53 & 13 \\
\hline 41 & Watts, Ray L & University of Alabama at Birmingham & 2,702 & 81 & 40 \\
\hline 42 & Braak, Heiko & University of Ulm & 2,672 & $\mathrm{n} / \mathrm{a}$ & $\mathrm{n} / \mathrm{a}$ \\
\hline 43 & Nutt, John G & Oregon Health \& Science University & 2,650 & 31 & -12 \\
\hline 44 & Beal, M Flint & Cornell University & 2,629 & 90 & 46 \\
\hline 45 & Larsen, Jan Petter & Stavanger University Hospital & 2,622 & 51 & 6 \\
\hline 46 & Rascol, Olivier & University of Toulouse & 2,607 & 32 & -14 \\
\hline 47 & Wszolek, Zbigniew K & Mayo Clinic - Florida & 2,584 & 73 & 26 \\
\hline 48 & Del Tredici K & University of Ulm & 2,558 & $\mathrm{n} / \mathrm{a}$ & $\mathrm{n} / \mathrm{a}$ \\
\hline 49 & Nussbaum, Robert L & University of California San Francisco & 2,542 & 38 & -11 \\
\hline 50 & Vila, Miquel & University Hospital Vall d'Hebron & 2,533 & 92 & 42 \\
\hline 51 & Oertel, Wolfgang $\mathrm{H}$ & The Philipps University & 2,525 & 45 & -6 \\
\hline 52 & Hattori, Nobutaka & Juntendo University School of Medicine & 2,500 & 24 & -28 \\
\hline 53 & Klein, Christine & University of Lübeck & 2,470 & $\mathrm{n} / \mathrm{a}$ & $\mathrm{n} / \mathrm{a}$ \\
\hline 54 & Tanner, Caroline M & The Parkinson's Institute & 2,378 & 19 & -35 \\
\hline 55 & Bonnet, Anne-Marie & Pitié - Salpêtrière University Hospital & 2,336 & 49 & -6 \\
\hline 56 & Isacson, Ole & Harvard University & 2,331 & $\mathrm{n} / \mathrm{a}$ & $\mathrm{n} / \mathrm{a}$ \\
\hline
\end{tabular}


Table 4 (continued)

\begin{tabular}{|c|c|c|c|c|c|}
\hline $\begin{array}{l}\text { Rank for } \\
\text { total } \\
\text { citations }\end{array}$ & Investigator & Affiliation & $\begin{array}{c}\text { Total } \\
\text { citations }\end{array}$ & $\begin{array}{l}\text { Table } 1 \\
\text { total } \\
\text { citations } \\
\text { rank }\end{array}$ & $\begin{array}{c}\text { Rise or fall } \\
\text { in Rank } \\
\text { from Table } 1 \\
\text { to Table } 4\end{array}$ \\
\hline$\overline{57}$ & Ondo, William G & Baylor College of Medicine & 2,330 & $\mathrm{n} / \mathrm{a}$ & $\mathrm{n} / \mathrm{a}$ \\
\hline 58 & Volkmann, Jens & Christian- Albrechts University & 2,323 & $\mathrm{n} / \mathrm{a}$ & $\mathrm{n} / \mathrm{a}$ \\
\hline 59 & Hirsch, Etienne C & Pitié - Salpêtrière University Hospital & 2,297 & 14 & -45 \\
\hline 60 & Barker, Roger A & University of Cambridge & 2,258 & $\mathrm{n} / \mathrm{a}$ & $\mathrm{n} / \mathrm{a}$ \\
\hline 61 & Healy, Daniel G & University College London & 2,256 & $\mathrm{n} / \mathrm{a}$ & $\mathrm{n} / \mathrm{a}$ \\
\hline 62 & Rüb, Udo & Goethe University & 2,242 & $\mathrm{n} / \mathrm{a}$ & $\mathrm{n} / \mathrm{a}$ \\
\hline 63 & Jenner, Peter & King's College London & 2,215 & 7 & -56 \\
\hline 64 & DeLong, Mahlon R & Emory University & 2,207 & 43 & -21 \\
\hline 65 & Deuschl, Günther & Christian-Albrechts University & 2,204 & $\mathrm{n} / \mathrm{a}$ & $\mathrm{n} / \mathrm{a}$ \\
\hline 66 & Shoulson, Ira & University of Rochester & 2,169 & 44 & -22 \\
\hline 67 & Abou-Sleiman, Patrick M & University College London & 2,153 & $\mathrm{n} / \mathrm{a}$ & $\mathrm{n} / \mathrm{a}$ \\
\hline 68 & Dickson, Dennis W & Mayo Clinic - Florida & 2,141 & $\mathrm{n} / \mathrm{a}$ & $\mathrm{n} / \mathrm{a}$ \\
\hline 69 & Trojanowski, John Q & University of Pennsylvania & 2,115 & 82 & 13 \\
\hline 70 & Stocchi, Fabrizio & IRCCS San Raffaele & 2,053 & $\mathrm{n} / \mathrm{a}$ & $\mathrm{n} / \mathrm{a}$ \\
\hline 71 & Oostra, Ben A & Erasmus University & 2,043 & $\mathrm{n} / \mathrm{a}$ & $\mathrm{n} / \mathrm{a}$ \\
\hline 72 & Burn, David J & Newcastle University & 2,035 & $\mathrm{n} / \mathrm{a}$ & $\mathrm{n} / \mathrm{a}$ \\
\hline 73 & Youdim, Moussa B H & Technion - Israel Institute of Technology & 2,009 & 25 & -47 \\
\hline 74 & Marek, Kenneth & Institute for Neurodegenerative Disorders & 1,975 & 76 & 3 \\
\hline 75 & de Vos, Rob A I & Laboratorium Pathologie Oost Nederland & 1,974 & $\mathrm{n} / \mathrm{a}$ & $\mathrm{n} / \mathrm{a}$ \\
\hline 76 & Giladi, Nir & Tel Aviv Sourasky Medical Center Sackler School of Medicine & 1,970 & $\mathrm{n} / \mathrm{a}$ & $\mathrm{n} / \mathrm{a}$ \\
\hline 77 & Shults, Clifford W & University of California San Diego & 1,935 & 89 & 13 \\
\hline 78 & Adler, Charles H & Mayo Clinic - Arizona & 1,934 & $\mathrm{n} / \mathrm{a}$ & $\mathrm{n} / \mathrm{a}$ \\
\hline 79 & Teismann, Peter & University of Aberdeen & 1,932 & $\mathrm{n} / \mathrm{a}$ & $\mathrm{n} / \mathrm{a}$ \\
\hline 80 & McKeith, Ian G & Newcastle University & 1,929 & $\mathrm{n} / \mathrm{a}$ & $\mathrm{n} / \mathrm{a}$ \\
\hline 81 & Berg, Daniela & University of Tübingen & 1,910 & $\mathrm{n} / \mathrm{a}$ & $\mathrm{n} / \mathrm{a}$ \\
\hline 82 & Hauser, Robert A & University of South Florida & 1,895 & 70 & -11 \\
\hline 83 & McNaught, Kevin St P & Mount Sinai School of Medicine & 1,884 & $\mathrm{n} / \mathrm{a}$ & $\mathrm{n} / \mathrm{a}$ \\
\hline 84 & Brown, Peter & University of Oxford & 1,862 & $\mathrm{n} / \mathrm{a}$ & $\mathrm{n} / \mathrm{a}$ \\
\hline 85 & Baptista, Melisa J & University of Sheffield & 1,854 & $\mathrm{n} / \mathrm{a}$ & $\mathrm{n} / \mathrm{a}$ \\
\hline 86 & Vitek, Jerrold L & University of Minnesota & 1,848 & $\mathrm{n} / \mathrm{a}$ & $\mathrm{n} / \mathrm{a}$ \\
\hline 87 & Meco, Giuseppe & Sapienza University & 1,823 & 88 & 1 \\
\hline 87 & Schapira, Anthony H V & University College London & 1,823 & 28 & -59 \\
\hline 89 & Björklund, Anders & Lund University & 1,810 & 23 & -66 \\
\hline 90 & Fraix, Valérie & Joseph Fourier University & 1,790 & $\mathrm{n} / \mathrm{a}$ & $\mathrm{n} / \mathrm{a}$ \\
\hline 91 & Lansbury, Peter T & Harvard University & 1,778 & 100 & 9 \\
\hline 92 & Greenamyre, J Timothy & University of Pittsburgh & 1,775 & 93 & 1 \\
\hline 93 & Tieu, Kim & University of Rochester & 1,758 & $\mathrm{n} / \mathrm{a}$ & $\mathrm{n} / \mathrm{a}$ \\
\hline 94 & Bentivoglio, Anna Rita & Università Cattolica del Sacro Cuore & 1,755 & $\mathrm{n} / \mathrm{a}$ & $\mathrm{n} / \mathrm{a}$ \\
\hline 95 & Gilks, William P & Trinity College - Dublin & 1,749 & $\mathrm{n} / \mathrm{a}$ & $\mathrm{n} / \mathrm{a}$ \\
\hline 96 & Schwarzschild, Michael A & Harvard University & 1,740 & $\mathrm{n} / \mathrm{a}$ & $\mathrm{n} / \mathrm{a}$ \\
\hline 97 & Sampaio, Cristina & Lisboa Medical University & 1,713 & $\mathrm{n} / \mathrm{a}$ & $\mathrm{n} / \mathrm{a}$ \\
\hline 98 & Vieregge, Peter & Clinic of Lippe-Lemgo & 1,690 & $\mathrm{n} / \mathrm{a}$ & $\mathrm{n} / \mathrm{a}$ \\
\hline 99 & Houeto, Jean-Luc & University of Poitiers & 1,677 & $\mathrm{n} / \mathrm{a}$ & $\mathrm{n} / \mathrm{a}$ \\
\hline 100 & Kulisevsky, Jaime & University of Barcelona & 1,676 & $\mathrm{n} / \mathrm{a}$ & $\mathrm{n} / \mathrm{a}$ \\
\hline
\end{tabular}

(n/a) indicates new names that appear in the most cited of the last 10 years who did not appear in the 25 year analysis

considered (as opposed to the default method of considering all citations regardless of the topic of the citing paper). By measuring only external citations, one can identify those researchers for whom the vast majority of citations come from outside the PD literature as opposed to those whose citations stem mainly from papers within the PD literature. We propose "broad impact" citations as a measure of the impact which a given investigator's PD work has outside PD research.

\section{Impact in the 21st century}

In order to get a sense for how the PD "impact landscape", might have changed in the 21 st century, a total-citations ranking was generated using only the subset of the papers from the original analysis published between 2001 and 2010. Employing the same methodology as described above, an article corpus was generated comprising 27,063 papers written in 
20 languages appearing in 1,740 journals by authors representing 97 countries.

\section{CONCLUSIONS}

Most names in Tables 1-3 will be clearly recognized by the majority of PD investigators as being "superstars" (for example the late David Marsden) in the PD community. By contrast, however, many of the investigators appearing in Table 4 are likely to be less well known. The appearance of such names in a top-100 $\mathrm{PD}$ ranking is evidence that there are a considerable number of "rising stars" who, in the last decade, have made significant contributions to the PD literature, often through molecular or genetic approaches. Given the more modern nature of their lines of investigation, these investigators are not nearly as prominent when studying a longer timeframe.

An interesting direction for future study that naturally follows the work presented in this paper would be a more in depth examination of those areas of basic science which have contributed heavily to the current understanding of PD. An example of this would be to attempt to quantify the degree to which genomics or studies of mitochondrial dysfunction and oxidative stress have inspired new lines of investigation within the PD research community.

\section{ACKNOWLEDGMENTS}

The authors would like to thank the referees for their insightful comments.

\section{REFERENCES}

[1] Ponce FA, \& Lozano AM (2011) The most cited works in Parkinson's disease. Movement Disorders 26(3), 380-390.

[2] Parkinson's Disease Top-20 Author Report 1996-2006 [Internet]: Thomson ISI; c2011 [cited 2011 5/2/2011]. Available from:http://www.esi-topics.com/parkinson/authors/b1 a.html

[3] Hirsch JE (2005) An index to quantify an individual's scientific research output. Proc Natl Acad Sci U S A 102(46), 16569-16572.

[4] Hirsch JE (2007) Does the H index have predictive power? Proc Natl Acad Sci U S A 104(49), 19193-19198.

[5] Spillantini MG, Schmidt ML, Lee VMY, Trojanowski JQ, Jakes R, \& Goedert M (1997) Alpha-synuclein in Lewy bodies. Nature 388(6645): 839-840.

[6] Mirra SS, Heyman A, McKeel D, Sumi SM, Crain BJ, Brownlee LM, Vogel FS, Hughes JP, van Belle G, \& Berg L (1991) The Consortium to Establish a Registry for Alzheimer's Disease (CERAD). Part II. Standardization of the neuropathologic assessment of Alzheimer's disease. Neurology 41(4): 479-486.

[7] Van Tol HH, Bunzow JR, Guan HC, Sunahara RK, Seeman P, Niznik HB, \& Civelli O (1991) Cloning of the gene for a human dopamine D4 receptor with high affinity for the antipsychotic clozapine. Nature 350(6319): 610-614.

[8] Sorensen AA (2009) Alzheimer's disease research: scientific productivity and impact of the top 100 investigators in the field. Journal of Alzheimer's Disease 16(3), 451-465.

[9] HistCite Product Features [Internet]: Thomson Reuters, Inc.; c2011 [cited 2011 5/2/2011]. Available from: http://thomson reuters.com/products_services/science/science_products/a-z/ histcite/\#tab2

[10] BiomedExperts Database [Internet]: Collexis, Inc.; c2011 [cited 2011 5/2/2011]. Available from: http://www.biomed experts.com/ 